\title{
Acoustic Properties of Porous Coatings for Hypersonic Boundary-Layer Control
}

\author{
Guillaume A. Brès* and Tim Colonius \\ California Institute of Technology, Pasadena, California 91125 \\ and \\ Alexander V. Fedorov主 \\ Moscow Institute of Physics and Technology, 140180, Zhukovsky, Moscow Region, Russia
}

DOI: $\underline{10.2514 / 1.40811}$

\begin{abstract}
Numerical simulations are performed to investigate the interaction of acoustic waves with an array of equally spaced two-dimensional microcavities on an otherwise flat plate without external boundary-layer flow. This acoustic scattering problem is important in the design of ultrasonic absorptive coatings for hypersonic laminar flow control. The reflection coefficient, characterizing the ratio of the reflected wave amplitude to the incident wave amplitude, is computed as a function of the acoustic wave frequency and angle of incidence, for coatings of different porosities, at various acoustic Reynolds numbers relevant to hypersonic flight. Overall, the numerical results validate predictions from existing theoretical modeling. In general, the amplitude of the reflection coefficient has local minima at some specific frequencies. A simple model to predict these frequencies is presented. The simulations also highlight the presence of resonant acoustic modes caused by coupling of small-scale scattered waves near the coating surface. Finally, the cavity depth and the porosity are identified as the most important parameters for coating design. Guidelines for the choice of these parameters are suggested.
\end{abstract}

\section{Nomenclature}

$\begin{array}{lll}\mathrm{AR} & =\text { cavity aspect ratio } \\ a & = & \text { speed of sound } \\ b & = & \text { cavity half-width } \\ \tilde{C} & = & \text { dynamic compressibility } \\ c & = & \text { phase speed } \\ c_{p} & =\text { specific heat at constant pressure } \\ f & = & \text { frequency } \\ H & = & \text { cavity depth } \\ k & = & \text { wave number } \\ m & = & \text { propagation constant } \\ P & = & \text { pressure } \\ P r & = & \text { Prandtl number } \\ \mathcal{R} & = & \text { reflection coefficient } \\ p & = & \text { acoustic pressure } \\ p_{i} & =\text { initial pulse amplitude } \\ R e & =\text { acoustic Reynolds number } \\ s & =\text { cavity spacing } \\ T & =\text { temperature } \\ t & = & \text { time } \\ x & = & \text { tangential direction } \\ y & = & \text { normal direction } \\ Z_{c} & =\text { characteristic impedance } \\ \alpha & = & \text { absorption coefficient } \\ \gamma & = & \text { specific heat ratio } \\ \delta_{\text {ent }} & =\text { entropy-layer thickness } \\ \delta_{S} & =\text { Stokes-layer thickness }\end{array}$

Presented as Paper 3903 at the 5th AIAA Theoretical Fluid Mechanics Conference, Seattle, WA, 23-26 June 2008; received 4 September 2008; revision received 12 February 2009; accepted for publication 2 February 2009. Copyright (C) 2009 by G.A. Brès, T. Colonius, and A.V. Fedorov. Published by the American Institute of Aeronautics and Astronautics, Inc., with permission. Copies of this paper may be made for personal or internal use, on condition that the copier pay the $\$ 10.00$ per-copy fee to the Copyright Clearance Center, Inc., 222 Rosewood Drive, Danvers, MA 01923; include the code 0001-1452/10 and $\$ 10.00$ in correspondence with the CCC.

${ }^{*}$ Currently Exa Corporation, Brisbane, CA 94005. Member AIAA.

${ }^{\dagger}$ Professor, Department of Mechanical Engineering, Mail Code 104-44. Member AIAA.

Frofessor, Department of Aeromechanics and Flight Engineering. Member AIAA.

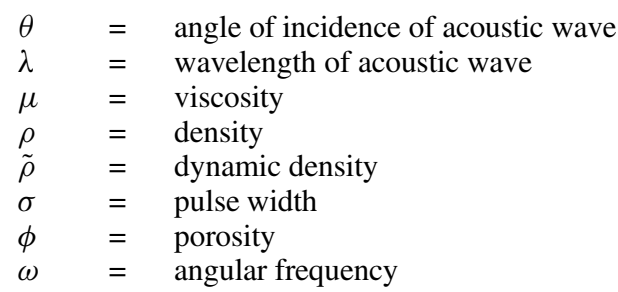

Subscript

$0=$ ambient property

Superscript

$* \quad=$ dimensional quantity

\section{Introduction}

$\mathbf{H}$ YPERSONIC laminar flow control for delayed laminarturbulent transition is an important component of economically viable hypersonic vehicles $[1,2]$. Wind-tunnel experiments and theoretical studies [3-8] have already demonstrated that an ultrasonically absorptive coating (UAC), which consists of a thin microporous layer, can suppress the second-mode instability and thereby delay transition of the predominately-two-dimensional boundary layer. Integration of this passive UAC device to thermal protection systems requires multidisciplinary efforts, including flow modeling, testing of materials and fabrication of prototypes. Among other issues, design and robust implementation of UAC on hypersonic vehicles hinges on the development of accurate and efficient models of the fundamental flow physics.

We report here on the first step in a broader computational study aimed at examining the detailed processes occurring on the scale of the microcavities that constitute the UAC and, in turn, lead to reduction of second-mode instability growth rates. We use direct numerical simulations (DNS) to investigate the interaction of incident acoustic waves with an array of equally spaced microcavities on a flat-plate surface without flow. Although the external boundarylayer flow is an important component of the UAC modeling, this unit problem is of particular interest because acoustic absorption represents the principle mechanism by which the UAC damps 
second-mode instabilities. Furthermore, the acoustic properties of UAC samples can be estimated in an economical way before testing in hypersonic wind tunnels, and theory and modeling are needed to design, implement, and interpret such experiments. To this end, we consider only acoustic waves interacting with the environment in an otherwise quiescent and uniform-temperature atmosphere. The effects of flow (and the resulting near-wall heating) will be reported in future publications.

We consider the reflection of plane monochromatic ultrasonic acoustic waves by a plane surface covered by a porous coating (Fig. 1). The coating has a regular structure (typical of UAC samples) consisting of equally spaced slots (2-D cavities). It is assumed that the UAC is absolutely rigid and its temperature is uniform and constant. The following is also assumed:

1) The cavity half-width $b$ and spacing $s$ satisfy the condition $b \sim s \ll \lambda$, where $\lambda$ is the wavelength of incident acoustic wave.

2) The cavity depth $H \sim \lambda$.

With these assumptions, the reflection coefficient (complex quantity characterizing the ratio of the reflected wave amplitude to the incident wave amplitude) is given by the relationship $[\underline{9}, \underline{10}]$

$$
\begin{gathered}
\mathcal{R}=\frac{Z_{c} \cos \theta-1}{Z_{c} \cos \theta+1}, \quad Z_{c}=\frac{Z_{c}^{*}}{\rho_{0} a_{0}}=\frac{1}{\rho_{0} a_{0} \phi} \sqrt{\frac{\tilde{\rho}}{\tilde{C}}} \operatorname{coth}(m H) \\
m=i \omega^{*} \sqrt{\tilde{\rho} \tilde{C}}
\end{gathered}
$$

Here, $Z_{c}$ is the normalized characteristic impedance and $m$ is the propagation constant of the porous layer, $\phi=2 b / s$ is the porosity, $\rho_{0}$ and $a_{0}$ are the density and speed of sound in ambient undisturbed gas, and $\omega^{*}=2 \pi f^{*}$ is the angular frequency of the acoustic wave. The dynamic density $\tilde{\rho}$, the dynamic compressibility $\tilde{C}$, and the propagation constant $m$, are calculated for an isolated deep cavity $(H \gg b)$ using the analytical solution derived by Kozlov et al. [11]. For the case of zero bulk viscosity and infinitesimal Knudsen number (ratio of the molecular mean free pass to the cavity half-width), this solution gives

$$
\tilde{\rho}=\frac{\rho_{0} \Lambda}{\Lambda-\tan \Lambda}, \quad \tilde{C}=\frac{1}{\gamma P_{0}}\left[1+(\gamma-1) \frac{\tan \tilde{\Lambda}}{\tilde{\Lambda}}\right]
$$

where $\Lambda=\sqrt{-i \omega^{*} \rho_{0} b^{2} / \mu_{0}}$ is proportional to the ratio of cavity half-width to the Stokes-layer thickness $\delta_{S}^{*}=\sqrt{2 \mu_{0} / \omega^{*} \rho_{0}}$. Here, $\tilde{\Lambda}=\sqrt{\operatorname{Pr}} \Lambda, \mu_{0}$ is the gas viscosity, $\gamma$ is the specific heat ratio, and $\operatorname{Pr}$ is the Prandtl number.

Note that this model accounts for viscous dissipation and heat conductivity inside individual cavities. However, end effects associated with scattering of incoming acoustic waves by the cavity mouth and local effects on the cavity bottom are ignored. Small-scale disturbances, which are generated near the UAC surface, are not

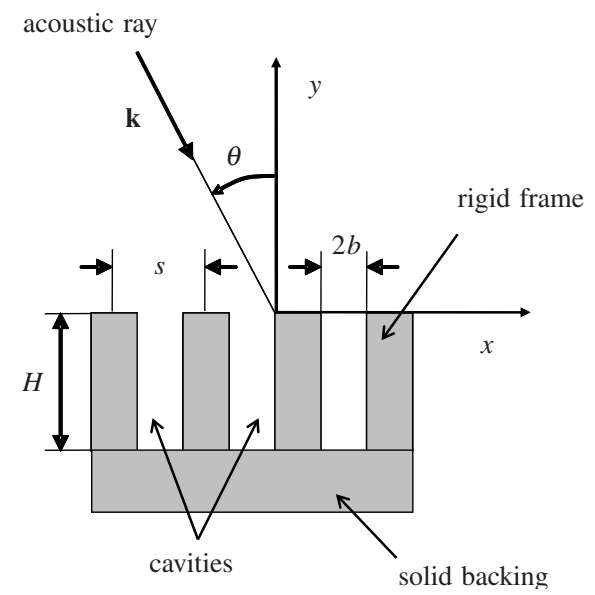

Fig. 1 Schematic of the reflection of acoustic wave from equally spaced 2-D cavities. considered. There is no coupling between disturbances in neighboring cavities, which may not be true for closely spaced cavities. This motivates us to perform DNS to clarify the aforementioned effects and validate the robustness of the theoretical model by comparisons with DNS solutions.

\section{Direct Numerical Simulations}

\section{A. Numerical Methods}

Brès and Colonius [12] developed an algorithm to solve the full compressible Navier-Stokes (NS) equations and study the flow over three-dimensional open cavities. The equations are solved for all of the scales of the flow without the use of any turbulence modeling. The code was modified to model the flow in individual cavities and in an array of equally spaced cavities in a rigid surface under a transitional boundary layer. We assume shock-free flow and use sixth-orderaccurate compact finite difference schemes for streamwise and normal directions and Fourier-spectral differentiation for homogeneous directions (when present). The usual compressible formulation is used to nondimensionalize the NS equations, where the superscript $*$ refers to the dimensional quantity, and the subscript 0 denotes the ambient undisturbed property:

$$
\begin{array}{crrl}
\rho=\frac{\rho^{*}}{\rho_{0}}, & P=\frac{P^{*}}{\rho_{0} a_{0}^{2}}, & T=\frac{T^{*} c_{p}}{a_{0}^{2}} \\
u_{i}=\frac{u_{i}^{*}}{a_{0}}, & x_{i}=\frac{x_{i}^{*}}{H}, & t=\frac{t^{*} a_{0}}{H}
\end{array}
$$

Regarding the properties of air, the assumptions are 1) continuum; 2) perfect gas with specific heat at constant pressure $c_{p}$, Prandtl number $\operatorname{Pr}=0.72$, and $\gamma=1.4$; and 3) Sutherland-law temperaturedependent viscosity/conductivity. These are appropriate for testing materials under laboratory conditions, but under flight conditions, the continuum assumption leads to some quantitative inaccuracies for very small pores, for which relevant Knudsen numbers can be as high as 0.4. This leads to less hydraulic resistance in the pores due to slip effects and has been analyzed in [11]. The temperature at the walls $T_{w}$ is assumed to be uniform and constant, such that $T_{w}=T_{0}$ in the case without external flow. The code can handle any type of block geometry (including the porous surface configuration shown in Fig. 1) and is fully parallelized using message-passing interface.

The current study focuses on the acoustic scattering by an array of equally spaced 2-D microcavities without external flow. The acoustic pressure fields $p=P-P_{0}$ generated by an initial acoustic disturbance (subscript $i c$ ) and its reflection (subscript ref) on a solid wall (without cavities) and on a porous wall (with cavities) are computed, and can be decomposed as

$$
\begin{aligned}
p_{\text {solid }}(x, y, t) & =p_{i c}(x, y, t)+p_{\text {ref,solid }}(x, y, t) \\
p_{\text {porous }}(x, y, t) & =p_{i c}(x, y, t)+p_{\text {ref,porous }}(x, y, t)
\end{aligned}
$$

We set the amplitude sufficiently small such that the resulting interaction is linear. Assuming that there is no overlapping of the initial and reflected waves, we can then identify the reflected signals at any fixed point $\left(x_{0}, y_{0}\right)$ and expand them into Fourier integrals:

$$
\begin{aligned}
p_{\text {ref,solid }}(t) & =\frac{1}{\sqrt{2 \pi}} \int_{-\infty}^{+\infty} \hat{p}_{\text {ref,solid }}(\omega) e^{-i \omega t} \mathrm{~d} \omega \\
p_{\text {ref,porous }}(t) & =\frac{1}{\sqrt{2 \pi}} \int_{-\infty}^{+\infty} \hat{p}_{\text {ref,porous }}(\omega) e^{-i \omega t} \mathrm{~d} \omega
\end{aligned}
$$

where $\omega=\omega^{*} H / a_{0}$. Because reflection from a solid wall has a reflection coefficient of 1 , we can calculate the complex reflection coefficient at $\left(x_{0}, y_{0}\right)$ (i.e., at angle $\left.\theta_{0}\right)$ for the porous wall $\mathcal{R}_{\mathrm{DNS}}(\omega)=$ $\hat{p}_{\text {ref,porous }}(\omega) / \hat{p}_{\text {ref,solid }}(\omega)$ and compare it with the theoretical value $\mathcal{R}(\omega)$ in Eq. (1).

\section{B. Computational Setup}

The configuration considered in this study corresponds to 2-D microcavities of constant length-to-depth ratio $\mathrm{AR}=2 b / H=0.12$. 

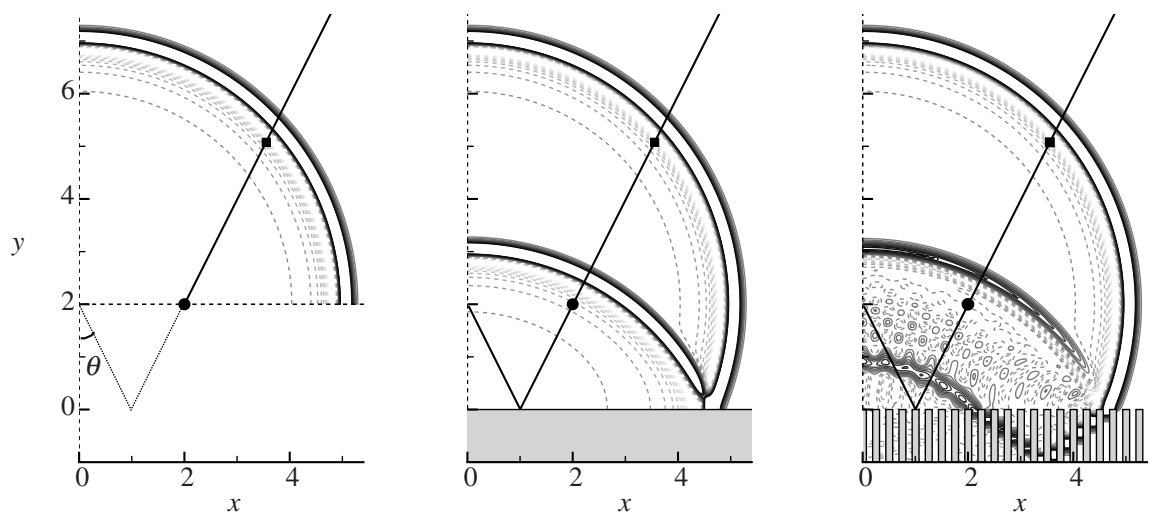

Fig. 2 Acoustic pressure field at time $t=10$ for simulations at $R e=100$ a) without wall, b) with solid wall, and c) with coating of porosity $\phi=0.48$. Twenty contours are shown between $p / p_{i}=-0.01$ and 0.01 , with negative contours dashed. For each angle $\theta$, the acoustic pressure field is recorded at $2 H$ (black circle), $5 \mathrm{H}$ (black square), and $10 \mathrm{H}$ along a ray above the wall. Symmetric boundary conditions are represented by the black dashed line.

This value matches the aspect ratio of the cylindrical cavities used in the experiment by Rasheed et al. [4].

In practice, the pressure field is measured at multiple locations (for a given height above the wall) to account for the dependence on $\theta$ (the angle of incidence of the acoustic wave) and at different heights (i.e., at $2 \mathrm{H}, 5 \mathrm{H}$, and $10 \mathrm{H}$ ) to quantify near-field effects (see Fig. 2). For all of the simulations, the initial disturbance at $t=0$ is a cylindrical acoustic pulse:

$$
\begin{aligned}
& p(x, y, 0)=\rho(x, y, 0)-\rho_{0}=p_{i} \exp \left[-\left(\left(x-x_{c}\right)^{2}\right.\right. \\
& \left.\left.\quad+\left(y-y_{c}\right)^{2}\right) / \sigma^{2}\right] \\
& u(x, y, 0)=v(x, y, 0)=0
\end{aligned}
$$

with $p_{i}=0.001$. Different widths $\sigma$ of the acoustic pulse were considered, effectively changing the frequency spectrum of the pulse. The width $\sigma=0.1$ was found to be adequate to accurately resolve frequencies up to $f=f^{*} H / a_{0} \approx 2$. For typical UAC parameters, this range of frequencies corresponds to the ultrasonicfrequency band and is sufficient to capture the frequency of the most amplified second-mode instability waves observed in experiments $[\underline{4}, \underline{13}]$ and numerical simulations $[\underline{3}, \underline{14}, \underline{15}]$.

To capture all of the successive reflected waves from the porous surface, the simulations are performed up to time $t=40$, until all of the disturbances in the flow have died away. To minimize the computational domain, the pulse is located at $\left(x_{c}, y_{c}\right)=(0,2)$ with symmetric boundary conditions at $x=x_{c}$. The grid extends up to $30 H$ and $18 H$ in the $x$ - and $y$ directions, respectively, with a large buffer zone at the top and right boundary, to avoid spurious reflections. For the configuration with the porous wall, the mesh contains about one and a half million grid points, with 12 and 100 points across each cavity length and depth, respectively.

Also, to ensure that the reflected waves are measured independently of the initial wave, an additional simulation without the wall (using symmetric boundary conditions at $y=y_{c}$ ) is performed to determine $p_{i c}(x, y, t)$. This particular procedure and choice of initial condition (rather than plane monochromatic acoustic waves) enables the computation of a complete mapping of the reflection coefficient $\mathcal{R}_{\text {DNS }}(\theta, \omega)$ in only three simulations (i.e., without wall, with solid wall, and with porous coating), as shown in Fig. 2.

\section{Validation}

All of the numerical simulations are performed on the same stretched Cartesian grid, with clustering of points near the walls to accurately capture the flow inside and around the pores. In particular, the interaction of acoustic waves with the solid wall leads to the formation of a Stokes layer and entropy layer on the wall surface. Their respective thicknesses can be estimated [16] as $\delta_{S}=$ $\delta_{S}^{*} / H=\sqrt{\mathrm{AR} /(\omega R e)}$ and $\delta_{\mathrm{ent}}=\delta_{S} / \sqrt{P r}$, where $R e=\rho_{0} a_{0} b / \mu_{0}$ is the acoustic Reynolds numbers based on the cavity half-width. These features are fully resolved on the chosen computational mesh, except for a high Reynolds number (e.g., $R e=1000$ ). However, for disturbances of ultrasonic frequency relevant to this study, these lengths are much smaller than the corresponding acoustic wavelength $\lambda=a_{0} / f^{*}$, and the Stokes and entropy layers can therefore be neglected (for further validation of this assumption and the numerical setup, see [17]).

Additionally, comparison of the reflection coefficients measured at $2 \mathrm{H}, 5 \mathrm{H}$, and $10 \mathrm{H}$ above the surface shows that near-field effects are negligible and that the reflection coefficient is independent of the height of the measurements [17]. In the remainder of the paper, the results will be presented for measurements at $2 \mathrm{H}$.

\section{Results and Discussion}

\section{A. Parametric Study}

The numerical simulations are performed at Reynolds numbers $R e=10,100$, and 1000 for coatings of porosity $\phi=0.2,0.48$, and 0.8 . These setup and flow conditions are chosen to enable future comparisons with ongoing experiments [13] on UAC samples. These values of $R e$ also correspond to the range of acoustics Reynolds numbers relevant for practical UAC (e.g., $b \approx 5$ to $100 \mu \mathrm{m}$ ) in highaltitude (e.g., $30 \mathrm{~km}$ ) hypersonic flight.

\section{Effect of Angle of Incidence}

Figure 3 shows the complete mapping of the reflection coefficient obtained for a coating of porosity $\phi=0.48$ at $R e=100$. This plot is representative of the results for the UAC of different porosities considered, and $R e \geq 100$. The reflection coefficient shows strong frequency modulations, largely independent of the angle of incidence, here up to $\theta \approx 60 \mathrm{deg}$. The physical source of these modulations is constructive/destructive interactions between reflections

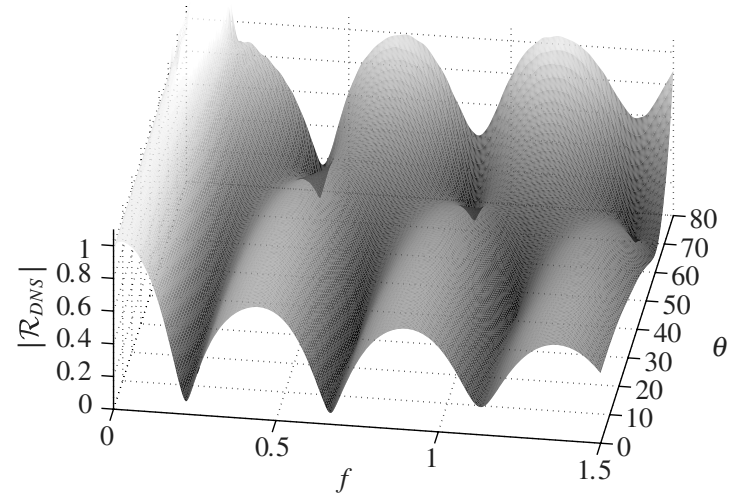

Fig. 3 Reflection coefficient amplitude $\left|\mathcal{R}_{\text {DNS }}\right|$ as a function of frequency $f$ and angle of incidence $\theta$ for a coating of porosity $\phi=0.48$ at $R e=100$. 
from the top solid wall and from the bottom of the porous surface and is discussed in detail in Sec. III.C.

As $\theta$ increases, an angle is reached at which the reflection coefficient is at its minimum value. This behavior is typical of the reflection of acoustic waves obliquely incident on a normally reacting surface (see Fig. 6.6.1 in [9]), for which the transmitted wave is refracted so that it propagates effectively only perpendicularly to the surface (i.e., inside the pores in our case). As $\theta$ goes to $90 \mathrm{deg}$, the theoretical reflection coefficient approaches $\mathcal{R}=-1$, and in this limit, the coating acts as a pressure-release surface. This result is confirmed by the DNS measurements, as shown in Fig. 4. At high angle of incidence, the ratio of peak pressure amplitude from UAC to

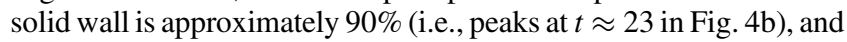
the waves are in opposition of phase, such that the acoustic pressure goes to zero at the top surface in the limit of $\theta=90 \mathrm{deg}$.

For second-mode instability waves, the angle of incidence can be estimated using a Wentzel-Kramers-Brillouin approximation [18]. In the inviscid limit, the pressure amplitude of the second mode is a solution of an eigenvalue problem (Eqs. 5-7 in [18]) and is expressed as a superposition of incident and reflected acoustic waves. The angle of incidence $\theta$ is estimated as

$$
\tan \left(\frac{\pi}{2}-\theta\right)=\lambda_{r}(0) / \alpha_{r} \approx \sqrt{\frac{M_{e}^{2}(U(0)-c)^{2}}{T(0)}-1}=\sqrt{\frac{M_{e}^{2} c^{2}}{T_{w}}-1}
$$

where $c=c^{*} / U_{e}$ is the phase velocity, $T_{w}=T_{w}^{*} / T_{e}^{*}$ is the wall temperature, and $M_{e}=U_{e} / a_{e}$ is the local Mach number. Here, the subscript $e$ denotes quantities at the upper boundary-layer edge. For the second mode at $M_{e} \geq 6$, the phase velocity is $c \approx 0.9$. For coldwall conditions relevant to experiment in shock tunnels $[4,19]$, the wall temperature is $T_{w} \approx 1$, which gives at high Mach number $\theta \approx 3-9 \mathrm{deg}$, close to $0 \mathrm{deg}$ (i.e., normal incidence). For moderate cooling [8] and an adiabatic wall [5], the estimated angle of incidence decreases to $\theta \approx 16$ and $26 \mathrm{deg}$, respectively. In all cases, the angle of incidence relevant for UAC design is smaller than $\theta=26 \mathrm{deg}$. Therefore, our analysis will mainly focus on the reflection coefficient at normal incidence.

\section{Effect of Viscosity}

Figure 5a shows the time history of the reflected signal $p_{\text {ref }} / p_{i}$ at normal incidence for a solid wall and a porous surface $(\phi=0.48)$ at the different Reynolds numbers considered $(R e=10,100$, and 1000). The main reflections from the top solid wall and from the bottom of the cavity can be identified at time $t \approx 4$ and 6 , respectively. The corresponding reflection coefficient is shown in Fig. 5b.

At higher Reynolds numbers $(R e=100$ and 1000), reflections from the bottom of the cavities lead to destructive/constructive reinforcement at some specific frequencies. These frequencies, corresponding to local minima and maxima of the reflection coefficient, are only weakly dependent on the Reynolds number. As viscosity is increased (e.g., $R e=10$ ), there is no additional reflection from the cavity bottom because of the increase in dissipation inside the pores, and the reflection coefficient monotonically decreases with frequency.

As discussed in [17], we suspect that the curvature of the wave front introduced some discrepancies at low frequencies, and our method overestimates the reflection coefficient for $f<0.1$. However, this limitation does not affect the range of ultrasonic frequency relevant for UAC design. Also, the additional pressure oscillations observed at late times (in particular, at $R e=1000$ and 100) correspond to the resonant modes discussed in Sec. III.D.

The influence of the viscosity can therefore be summarized qualitatively as follows: there is a critical Reynolds number above which acoustic disturbances are not completely absorbed inside the pores. In that case, interference between incoming and outgoing (reflected from the cavity bottom) waves leads to significant decrease of the reflection coefficient at specific frequencies. A simple model for the prediction of these frequencies is presented in Sec. III.C.

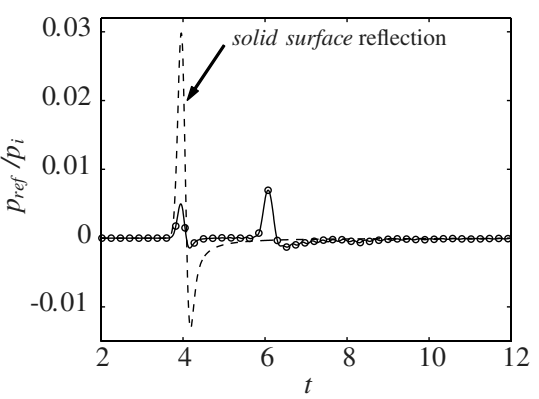

a)

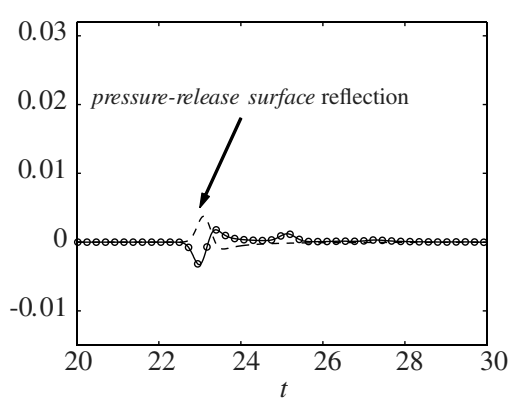

b)

Fig. 4 Pressure-time history at $2 \mathrm{H}$ above the top surface and $\mathrm{Re}=100$ for the solid wall (dashed line) and UAC of porosity $\phi=0.8$ (circle) at angle of incidence a) $\theta=0 \mathrm{deg}$ and b) $\theta=80 \mathrm{deg}$.

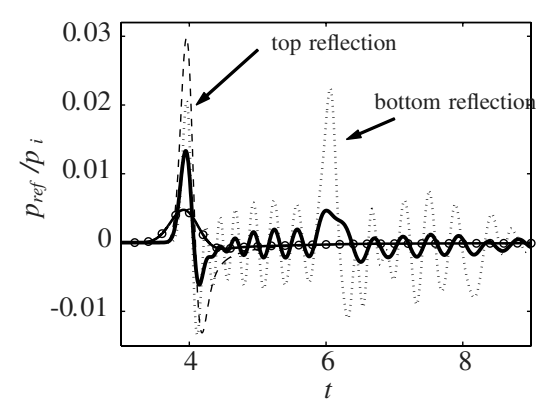

a)

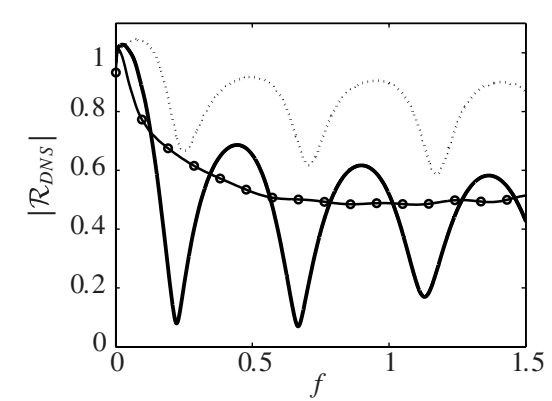

b)

Fig. 5 Effect of viscosity on the reflection of the acoustic wave from a coating of porosity $\phi=0.48$ at normal incidence $(\theta=0 \mathrm{deg}) ; \boldsymbol{R}=10$ (circle), $R e=100$ (solid line), and $R e=1000$ (dotted line): a) pressure-time history at $2 H$ above the top surface (for comparison, the reflected signal from the solid wall at $R e=100$ is also presented) (dashed line) and b) reflection coefficient amplitude. 


\section{Effect of Porosity}

To investigate the influence of porosity, simulations are performed for $\phi=0.2,0.48$, and 0.8 , keeping the same cavity aspect ratio. The pressure-time history and reflection coefficient at normal incidence for $R e=100$ are shown in Figs. 6a and 6b, respectively. As expected, the amplitude of the reflection from the top surface (i.e., the peak at $t \approx 4$ in Fig. 6a) decreases with porosity, whereas the reflection from the bottom of the cavity (i.e., the peak at $t \approx 6$ ) increases. The frequencies corresponding to local minima and maxima of the reflection coefficient are largely unaffected by the change in porosity. In contrast, the amplitude of the reflection coefficient increases as porosity decreases. This result is consistent with the limit value $|\mathcal{R}|=1$ for $\phi=0$, and with the observations that UAC performance increases with porosity. The porosity is therefore a critical parameter for the scattering and absorption of acoustic waves by a porous surface, and this feature is further discussed in Sec. III.E.

As mentioned previously, the additional oscillations observed in the pressure-time history correspond to a resonant mode. From Fig. 6a, it is clear that the oscillation frequency and amplitude depend on porosity. In particular, for $\phi=0.2$, the presence of the resonant mode of frequency approximately 0.83 strongly affects the calculation of the reflection coefficient, and the data are not reliable for $f \geq 0.8$. Therefore, the results are not shown in Fig. $6 \mathrm{~b}$. The resonant mode is examined in more detail in Sec. III.D.

\section{B. Comparison of DNS with Theory}

The comparisons between the reflection coefficient obtained from DNS and from theory at $R e=100$ are presented in Figs. 7a-7c for porosity $\phi=0.8,0.48$, and 0.2 , respectively. Overall, there is good agreement between the DNS results and the theoretical predictions, with less than $5 \%$ error in most cases. The formulation described in Sec. I accurately captures the dependence of the reflected acoustic waves on the angle of incidence, frequency, and porosity. As expected, the Stokes and entropy layers on the cavity mouth and bottom, which are resolved in the DNS but not accounted for in the theoretical model, have little influence on the reflection of ultrasonic acoustic waves.

In particular, the theory confirms that the reflection coefficient is approximately independent of the angle of incidence for $\theta \leq 30$ $35 \mathrm{deg}$. There is also good agreement on the specific frequencies of minimum reflection coefficient, for both low and high angles of incidence. The pressure-time history at $\theta=0$ and 80 deg in Fig. 4 gives some insight on the dependence of these frequencies on the angle of incidence. At normal incidence in Fig. 4a, the reflected wave from the top surface of the porous wall is in phase with the reflected wave from the solid surface, but approximately in opposition of phase at $\theta=80 \mathrm{deg}$ in Fig. $4 \mathrm{~b}$. This observation suggests that the specific values of the frequencies of minimum reflection coefficient are related to the phase between the reflected waves, which is the starting point of the analysis presented in Sec. III.C.

Detailed comparison between theory and DNS results at normal incidence is shown in Fig. 8. For all of the porosities and Reynolds numbers considered in this study, the theoretical predictions match the DNS measurements well (in particular, at low frequency), with less than $2 \%$ error. The agreement is good even for high porosity, in which neighboring pores are closely spaced and the theory neglects the coupling of disturbances. The discrepancy increases with frequency, most likely because the theory assumes that the acoustic wavelength $\lambda$ is much larger than the cavity half-width $b$ and the spacing $s$. For instance, for $f=1.5$ and porosity $\phi=0.48$, the ratio $s / \lambda \approx 0.4$, and the error increases to about $5 \%$. Also, for a lower Reynolds number (i.e., $R e=10$ ), the viscous effects lead to significant absorption and dispersion of the incoming wave. In this case, the error is greater than $10 \%$ for $f \geq 0.75$, and a smaller initial pulse width $\sigma$ may be required to fully capture the reflection coefficient at high frequency and better match the theoretical predictions.

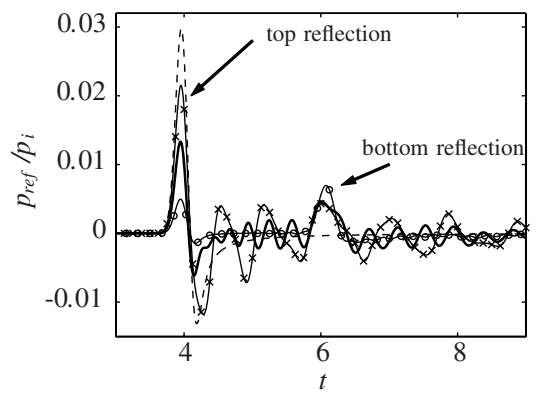

a)

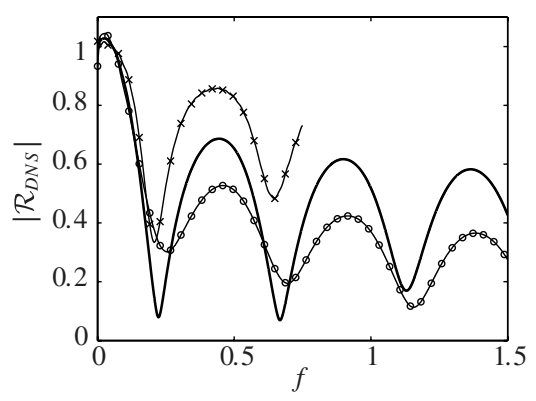

b)

Fig. 6 Effect of porosity on the reflection of acoustic wave from porous surfaces at $R e=100$ and normal incidence $(\theta=0 \mathrm{deg})$ for porosity $\phi=0.2(\mathrm{x})$, $\phi=0.48$ (solid line), and $\phi=0.8$ (circle): a) pressure-time history at $2 \mathrm{H}$ above the top surface (for comparison, the reflected signal from the solid wall is also presented) (dashed line) and $b$ ) reflection coefficient amplitude.

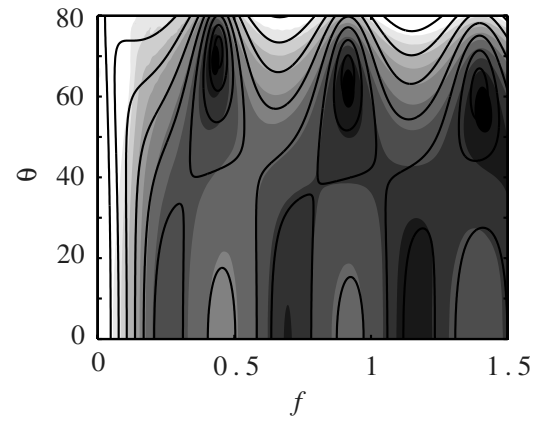

a)

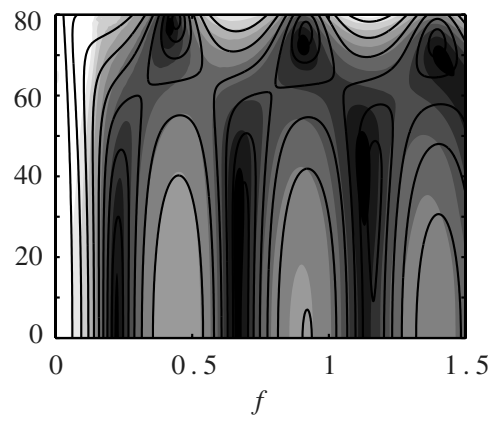

b)

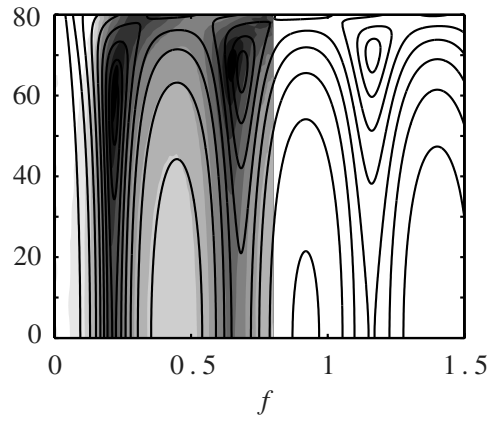

c)

Fig. 7 Comparison of the reflection coefficient amplitude from DNS (flooded contours) and theory (solid line) at $\operatorname{Re}=100$ for porosity a) $\phi=0.8$, b) $\phi=0.48$, and c) $\phi=0.2$. Ten contours are shown between $|\mathcal{R}|=0$ (black) and 1 (white). 


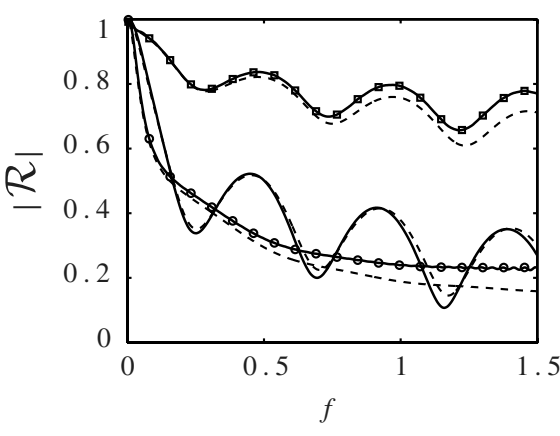

a)

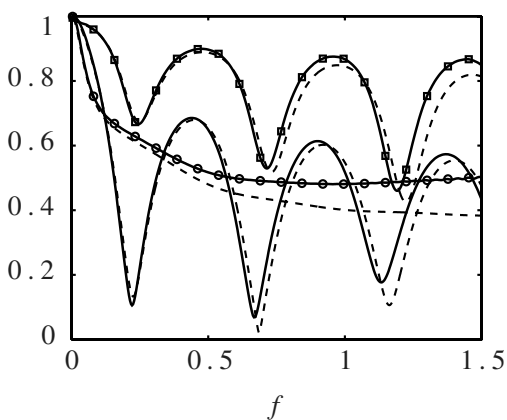

b)

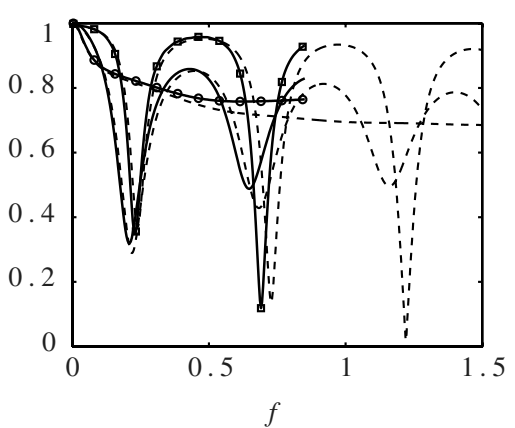

c) $R e=100$ (solid line), and $\operatorname{Re}=1000$ (square) for porosity a) $\phi=0.8, b) \phi=0.48$, and c) $\phi=0.2$.

Note that for the low-porosity case in Figs. 7c and 8c, the DNS results are affected by the resonant mode for $\bar{f}>0.85$ and are not shown. This resonant mode is not captured in the theoretical model because it originates from small-scale disturbances generated near the UAC surface and coupling between neighboring cavities, as discussed in Sec. III.D.

\section{Estimate of the Specific Frequencies of Minimum Reflection}

We present here a simple model to predict the frequencies of minimum reflection coefficient. For a plane monochromatic wave traveling in the $+y$ direction (i.e., reflected wave from the solid wall), the solution to the wave equation is of the form $p_{1} \exp [i(\omega t-k y)]$. Similarly, the reflected wave from the porous surface is of the form $p_{2} \exp [i(\omega t-k(y+\Delta y))]$, where $\Delta y=2 H$ corresponds to the distance traveled by the wave inside the pores. Minima of the reflection coefficient are obtained when the reflection from the solid wall and the porous surface are in opposition of phase: that is, $k_{r} \Delta y=(2 n-1) \pi$, where $k_{r}$ is the real part of the wave number $k=\omega^{*} / a_{0}$, and $n=1,2,3$, etc. In the inviscid approximation, the wave number in dimensional form is $k_{r}=k=2 \pi f^{*} / a_{0}$, and the frequencies of minimum reflection coefficient relevant for UAC are estimated as

$$
\frac{f^{*} H}{a_{0}}=f_{n}=(2 n-1) / 4
$$

where $n=1,2$, and 3 lead to the specific frequencies $f_{1}=0.25$, $f_{2}=0.75$, and $f_{3}=1.25$, respectively.

The effect of viscosity can also be estimated using the absorption coefficient $\alpha_{w}^{*}$ for wall losses in wide pipes [9]. This approximation is valid for pores that are wide compared with the Stokes-layer thickness (i.e., $b / \delta_{S}^{*} \gg 1$, which is approximately the case in our study for $R e \geq 100$. The presence of the viscous boundary layer modifies the wave speed $c_{w}^{*}$ of the acoustic wave such that $c_{w}=c_{w}^{*} / a_{0} \approx 1-\alpha_{w}^{*} / k$. In that case, $k_{r}=\omega^{*} / c_{w}^{*}=k / c_{w}$, and the frequencies of minimum reflection coefficient are now the solution of the equation

$$
\frac{f_{n}}{c_{w}\left(f_{n}\right)}=(2 n-1) / 4 \quad n=1,2,3
$$

The predicted analytical frequencies for $R e=100, R e=1000$, and the inviscid limit are compared with DNS and theory in Fig. 9. The results are presented for the different porosities considered, which lead to some scattering of the data. Overall, there is a reasonable agreement between the estimations and the measured frequencies of minimum reflection, with approximately less than $10 \%$ error. The formulation with viscosity effects accurately captures the Reynolds number dependence of these frequencies: namely, that they decrease with viscosity. Although the prediction at $R e=1000$ provides good results, the decrease in frequency at $R e=100$ is overestimated, most likely because the assumption that $b / \delta_{S}^{*} \gg 1$ starts to break down.
This model is based on the assumption that the phase difference between the reflected waves is introduced only by the traveled distance in the pores. As mentioned in Sec. III.A.1, an additional phase shift is observed at high angle of incidence. In the inviscid approximation, if the waves are initially in opposition of phase (as in Fig. 4b), then the frequencies of minimum reflection coefficient are now $f=n / 2$, where $n=1,2$, and 3. These estimates are in good agreement with the DNS and theoretical results for $\theta>70$ deg in Figs. $7 \mathrm{a}$ and $7 \mathrm{~b}$. Also note that a similar condition can be derived to predict the frequencies of maximum reflection coefficient.

\section{Resonant Modes}

In most of our simulations, we observe the presence of additional oscillations in the flowfield, with a frequency strongly dependent on porosity. We argue here that these oscillations correspond to resonant modes caused by small-scale disturbances generated near the UAC surface and coupling between neighboring cavities. In this context, the term "resonant" is used to qualify the mode frequencies in a broad sense, even though the oscillations are actually slowly damped and decay in time.

In this mechanism, the interaction of the acoustic wave with the porous surface generates scattered waves at the pore corners. These waves created near each cavity mouth are coupled with those from the neighboring pores and lead to oscillations in the flowfield above the porous surface. As these acoustic disturbances travel back and forth between pores, the wavelength of the oscillations $\lambda_{\text {res }}$ is related to the spacing between cavities by $\lambda_{\text {res }}=s$. The nondimensionalized frequency of the resonant mode is then estimated as

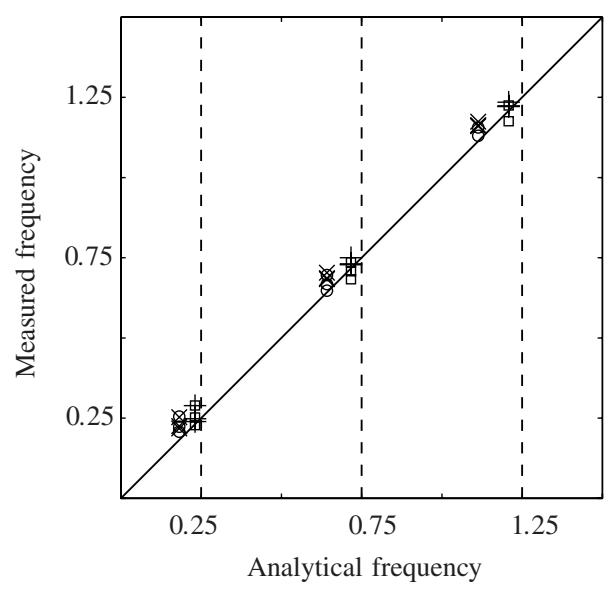

Fig. 9 Comparison of the analytical frequency predicted from Eq. (7) with frequencies of minimum reflection measured from DNS $(\bigcirc R e=$ 100 and $\square R e=1000)$ and theory $(\times R e=100$ and $+R e=1000)$. The solid line has unit slope and indicates exact correlation between analytical and measured frequencies. The inviscid estimate from Eq. (6) is shown by the dashed line. 


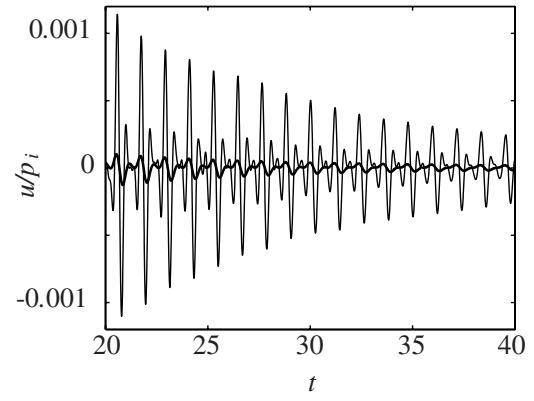

a)

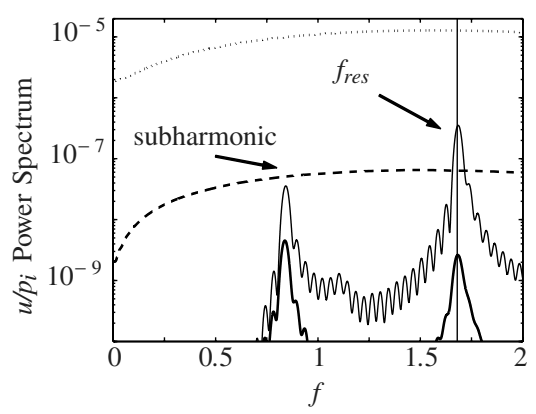

b)

Fig. 10 Resonant mode for a UAC of porosity $\phi=0.2$ at $R e=100$ (solid line) and $R e=1000$ (thick line): a) velocity-time history and b) corresponding power spectrum. The power spectrum of the full signal without wall (dotted line) and with solid surface (dashed line) are also shown, for $R e=1000$. The vertical line corresponds to the resonant frequency predicted with Eq. (

$$
f_{\text {res }}=\frac{H}{\lambda_{\text {res }}}=\frac{\phi}{A r}
$$

Figure 10 a shows a portion of the time history of the tangential velocity $u$ at $\theta=10 \mathrm{deg}$ and $2 H$ above a coating of porosity $\phi=0.2$ at $R e=100$ and 1000. At that location, the reflections of the acoustic pulse are measured earlier in time. Consequently, for $t \geq 20$, the resonant mode is the only unsteady feature of the flow. Similar oscillations, largely independent of location on the computational grid, are observed for the other flowfield variables. As expected, the oscillation amplitude of the acoustic resonant modes decreases with Reynolds number because of viscous absorption, and for a lower Reynolds number (i.e., $R e=10$ ), the oscillations are completely damped. The corresponding power spectrum is shown in Fig. 10b, and the predicted resonant frequency from Eq. (8) (represented by the vertical line) is in excellent agreement with the DNS measurement. We also observe a significant contribution from the first subharmonic of frequency $f_{\text {res }} / 2$ (i.e., of wavelength $2 s$ ), which corresponds to interaction between cavities one pore away from each other.

To quantify the relevance of the resonant mode compared with the other acoustic reflections, the power spectrum of the full time signal without wall and from the reflection off the solid surface are also presented in Fig. 10b. At the resonant frequency and subharmonic, the energy content of the solid-wall reflection and the resonant mode are similar for $R e=1000$, and the resonant frequencies are less energetic at $R e=100$ because of viscous absorption.

Similar agreement between the measured and predicted resonant frequencies are obtained for porosities $\phi=0.48$ and 0.8 , with less than $2 \%$ error. In this mechanism, the resonant frequency depends on the cavity aspect ratio (constant in our study) and is proportional to porosity. Therefore, for coatings of high porosity, which are of interest for laminar-flow-control applications, these frequencies are higher than the ultrasonic-frequency band relevant for UAC and do not affect the computation of the reflection coefficients. However, the resonant disturbances may interact with the boundary-layer flow and cause detrimental tripping effect. This issue should be addressed in future work with the presence of the outer boundary-layer flow.

\section{E. Guidelines for UAC Design}

For the 2-D microcavities considered in this study, the first important parameter is the cavity depth. In the range of acoustic Reynolds numbers relevant for practical UAC, reflections from the bottom of the cavities lead to minima of the reflection coefficient at some specific frequencies. Using the estimations from Eqs. (6) and (7), the cavity depth $H$ could be chosen so that the predicted frequency of minimum reflection matches the frequency of the most amplified second-mode instability waves observed in experiments. Because our results show that this mechanism is only weakly affected by the porosity, the parameter $H$ could be tuned up independently of the parameter $\phi$. Although the length-to-depth ratio of the pores was kept constant in our study, matching typical values from experiments, more simulations are underway to examine in details the effects of the cavity-aspect-ratio parameter.
Porosity is the other critical parameter for UAC design. Previous numerical simulations [3] have suggested that UAC performance increases with porosity, and our parametric study tends to confirm this result. Overall, the amplitude of the reflection coefficient decreases with higher porosity, as the scattering and absorption of acoustic waves by the UAC is enhanced. Furthermore, for high porosity, the resonant acoustic mode has a frequency much higher than the ultrasonic-frequency band relevant for UAC and is not expected to significantly affect the performance. More work is required to investigate these acoustic modes (in particular, for threedimensional pores).

In theory, the most promising configurations correspond to square, rectangular, and honeycomb 3-D patterns, with porosity up to 70$90 \%$ (see [19]). For such geometries, high-fidelity simulations are required to accurately describe the interactions between closely spaced pores. Additional work might also be needed to formulate and experimentally verify the structural integrity of these high-porosity coatings. Future studies will therefore focus on extending the current UAC research to realistic three-dimensional high-porosity coatings, with circular cavities, rectangular pores, or more complex geometry.

Finally, our DNS results show that the theoretical model presented in Sec. I is a robust and accurate tool for UAC design. The theoretical prediction of the reflection coefficient also agrees well with benchmark (no flow) measurement [20] conducted for a UAC of regular microstructure at various ambient pressures, with emphasis on low pressures relevant to high-altitude hypersonic flights. This set of theoretical, numerical, and experimental tools can be valuable to estimate, in an economical way, the acoustic properties of UAC samples before their testing in hypersonic wind tunnels.

\section{Conclusions}

The interaction of incident acoustic waves with an array of equally spaced microcavities on a flat-plate surface was investigated using theoretical modeling and direct numerical simulations. Because the second-mode instability of hypersonic boundary layer represents trapped acoustic waves of the ultrasonic-frequency band, it is assumed that basic features of its interaction with porous coating can be captured by considering this acoustic scattering problem with no external flow.

The simulations were performed for a porous coating consisting of 2-D cavities of constant length-to-depth ratio, with an incoming cylindrical acoustic pulse as the initial condition. The reflection coefficient was computed as a function of the acoustic wave frequency and angle of incidence, for coatings of different porosity, at various Reynolds numbers relevant to hypersonic flight. Comparisons with theoretical prediction showed excellent agreement with the DNS results in the parametric range relevant to UAC applications for laminar flow control. Overall, the reflection off the UAC decreases with higher porosity, and in most cases, minima of the reflection coefficient exist at some specific frequencies. A model to predict these frequencies was proposed and showed good agreement with the numerical and theoretical data. 
The simulations also highlighted the presence near the UAC surface of resonant acoustic modes caused by the coupling of smallscale scattered waves generated by neighboring pores. A formulation to estimate the resonant frequency was presented, and the predicted frequencies agreed well with DNS measurements. Although the resonant modes were not captured by the theoretical model, they are mainly relevant for coatings with cavity spacing of the same order than the wavelength of the incident wave. In practice, typical UAC for laminar-flow-control applications has spacing less than 0.1 of the second-mode wavelength instability; that is, the resonant frequency is much larger than the second-mode frequency. Nevertheless, the resonant acoustic modes may be excited by high-frequency disturbances of the outer flow and trip the boundary layer, similar to smallscale distributed roughness. The feasibility of this detrimental effect should be addressed in future numerical simulations including the outer flow.

Finally, based on our parametric study of the geometrical factors and flow conditions effects, we identified the cavity depth $H$ and the porosity $\phi$ as the most important parameters for UAC design. Guidelines for the choice of these parameters were also suggested. It is our hope that a better understanding of these acoustic properties will lead to improvements in existing UAC models.

\section{Acknowledgments}

This work was supported by the U.S. Air Force Office of Scientific Research under contract FA9550-06-C-0097. The authors would like to acknowledge contributions to this work by Norm Malmuth, whose passionate work on diverse topics, including ultrasonically absorptive coating, and generosity to his collaborators will be sorely missed.

\section{References}

[1] Malik, M. R., Zang, T. A., and Bushnell, D. M., "Boundary Layer Transition in Hypersonic Flows," AIAA Paper 90-5232, 1990.

[2] Kimmel, R., "Aspects of Hypersonic Boundary Layer Transition Control," AIAA Paper 2003-0772, 2003.

[3] Fedorov, A. V., Malmuth, N. D., Rasheed, A., and Hornung, H. G., "Stabilization of Hypersonic Boundary Layers by Porous Coatings," AIAA Journal, Vol. 39, No. 4, 2001, pp. 605-610. doi: $10.2514 / 2.1382$

[4] Rasheed, A., Hornung, H. G., Fedorov, A. V., and Malmuth, N. D., "Experiments on Passive Hypervelocity Boundary Layer Control Using an Ultrasonically Absorptive Surface," AIAA Journal, Vol. 40, No. 3, 2002, pp. 481-489.

doi: $10.2514 / 2.1671$

[5] Fedorov, A. V., Shiplyuk, A. N., Maslov, A. A., Burov, E. V., and Malmuth, N. D., "Stabilization of a Hypersonic Boundary Layer Using and Ultrasonically Absorptive Coating," Journal of Fluid Mechanics, Vol. 479, 2003, pp. 99-124. doi: $10.1017 / \mathrm{S} 0022112002003440$

[6] Fedorov, A. V., Kozlov, V. F., Shiplyuk, A. N., Maslov, A. A., Sidorenko, A. A., Burov, E. V., and Malmuth, N. D., "Stability of Hypersonic Boundary Layer on Porous Wall with Regular Microstructure," AIAA Paper 2003-4147, 2003.

[7] Bountin, D., Shiplyuk, A., Maslov, A., and Chokani, N., "Nonlinear Aspects of Hypersonic Boundary Layer Stability on a Porous Surface," AIAA Paper 2004-0255, 2004.

[8] Maslov, A. A., Shiplyuk, A. N., Sidorenko, A. A., Polivanov, P., Fedorov, A. V., Kozlov, V. F., and Malmuth, N. D., "Hypersonic Laminar Flow Control Using a Porous Coating of Random Microstructure," AIAA Paper 2006-1112, 2006.

[9] Kinsler, L. E., Frey, A. R., Coppens, A. B., and Sanders, J. V., Fundamentals of Acoustics, 4th ed., Wiley, New York, 2000.

[10] Lependin, L. F., Acoustics, Vysshaya Shkola, Moscow, 1978.

[11] Kozlov, V. F., Fedorov, A. V., and Malmuth, N. D., "Acoustic Properties of Rarefied Gases Insides Pores of Simple Geometries," Journal of the Acoustical Society of America, Vol. 117, No. 6, 2005, pp. 3402-3412. doi:10.1121/1.1893428

[12] Brès, G. A., and Colonius, T., "Three-Dimensional Instabilities in Compressible Flow over Open Cavities," Journal of Fluid Mechanics, Vol. 599, Mar. 2008, pp. 309-339. doi:10.1017/S0022112007009925

[13] Stetson, K. F., Thomson, E. R., Donaldson, J. C., and Siler, L. G., "Laminar Boundary-Layer Stability Experiments on a Cone at Mach 8, Part 1: Sharp Cone," AIAA Paper 83-1761, 1983.

[14] Egorov, I. V., Fedorov, A. V., Novikov, A. V., and Soudakov, V. G., "Direct Numerical Simulation of Supersonic Boundary-Layer Stabilization by Porous Coatings," AIAA Paper 2007-948, 2007.

[15] Brès, G. A., Colonius, T., and Fedorov, A. V., "Stability of Temporally Evolving Supersonic Boundary Layers Over Micro-Cavities for Ultrasonic Absorptive Coatings," AIAA Paper 2008-4337, 2008.

[16] Pierce, A. D., Acoustics: An Introduction to Its Physical Principles and Applications, McGraw-Hill, New York, 1989.

[17] Brès, G. A., Colonius, T., and Fedorov, A. V., "Interaction of Acoustic Disturbances with Micro-Cavities for Ultrasonic Absorptive Coatings," AIAA Paper 2008-3903, 2008.

[18] Malmuth, N. D., Fedorov, A. V., Shalaev, V., Cole, J., Khokhlov, A., Hites, M., and Williams, D., "Problems in High Speed Flow Prediction Relevant to Control," AIAA Paper 98-2695, 1998.

[19] Fedorov, A. V., and Malmuth, N. D., "Parametric Studies of Hypersonic Laminar Flow Control Using a Porous Coating of Regular Microstructure," AIAA Paper 2008-588, 2008.

[20] Fedorov, A. V., Kozlov, V. F., and Addison, R. C., "Reflection of Acoustic Disturbances from a Porous Coating of Regular Microstructure," AIAA Paper 2008-3902, 2008. 